\title{
THE ORNITHOLOGY OF THE REAL EXPEDICIÓN BOTÁNICA A NUEVA ESPAÑ (1787-1803): AN ANALYSIS OF THE MANUSCRIPTS OF JOSÉ MARIANO MOCIÑO
}

\author{
Adolfo G. Navarro-Sigüenza ${ }^{1,5}$, A. Townsend Peterson ${ }^{2}$, Miguel Ángel $^{3}$ \\ PUIG-SAMPER $^{3}$, AND GRACIELA ZAMUDIO ${ }^{4}$ \\ ${ }^{1}$ Museo de Zoología, Facultad de Ciencias, Universidad Nacional Autónoma de México, A.P. 70-399, México, \\ D.F. 04510 México \\ ${ }^{2}$ Natural History Museum and Biodiversity Research Center, University of Kansas, Lawrence, Kansas 66045 \\ ${ }^{3}$ Instituto de Historia, Consejo Superior de Investigaciones Cientificas (CSIC), Duque de Medinaceli No. 6, \\ 28014 Madrid, Spain \\ ${ }^{4}$ Laboratorio de Historia de la Biología, Facultad de Ciencias, Universidad Nacional Autónoma de México, \\ México, D.F. 04510 México
}

\begin{abstract}
Until recently, a major gap has existed in our knowledge regarding Mexican bird information from the Real Expedición Botánica a Nueva España in the late 1700s. This expedition (1787-1803) was commanded by Martín de Sessé; the Mexican scientist José Mariano Mociño joined the group in 1790, but his ornithological findings were never published and have long been considered lost. However, study of the Sessé-Mociño ornithological results began in 1979 with the appearance of a small collection of original paintings, apparently from the expedition. Later, in 1997, unpublished manuscripts were discovered in the library of the Museo Nacional de Ciencias Naturales, Madrid that included descriptions of many bird species, again apparently Sessé-Mociño material. These discoveries, covering an important portion of the overall ornithological results of the expedition, make possible a broader study of the Sessé-Mociño ornithology: a taxonomic list using modern nomenclature, an analysis of the correspondence between the paintings and the manuscripts, and conclusions regarding the provenance of those materials. Of a total of 83 paintings available, we were able to identify 78 to species, and 5 only to family. In the manuscripts, 290 species were treated, but for 27 , the descriptions were fragmentary and insufficient for identification; of the remaining 263 species, we arrived at a species-level identification for 242, and identified the remainder to genus (19) or family (2). The recent discovery of these ornithological texts and paintings offers a unique view of the history of ornithology as well as of the environmental history of Mexico.
\end{abstract}

Key words: eighteenth century, Mexico, Mociño, ornithology, Sessé.

La Ornitología de la Real Expedición Botánica a Nueva España (1787-1803): un análisis de los manuscritos de José Mariano Mociño

Resumen. Hasta hace poco, existía un gran hueco en el conocimiento de las aves estudiadas por los miembros de la Real Expedición Botánica a Nueva España, comandada por Martín de Sessé, que se llevó a cabo entre 1787 y 1803, a la que se incorporó el científico mexicano José Mariano Mociño en 1790. El estudio de los resultados ornitológicos empieza a partir de que una colección de láminas originales realizadas por los pintores de la expedición a Nueva España fue identificada en Barcelona, España, en 1979. Posteriormente, en 1997, se localizaron en el Museo Nacional de Ciencias Naturales de Madrid, los manuscritos inéditos corresponientes a las descripciones de numerosas especies de aves procedentes de la expedición. El contar ahora con las láminas y los manuscritos, nos permite presentar una lista taxonómica, desde el punto de vista moderno, de las aves descritas en esos manuscritos y láminas y analizar si existe una correspondencia entre ambas fuentes de información. Un total de 83 láminas estuvieron disponibles, de éstas se logró identificar a todas hasta algun nivel taxonómico; de ellas 78 fueron identificadas a nivel de especie y 5 solamente a familia. En los manuscritos se encontraron a 290 especies, de las cuales 27 contenían una descripción demasiado breve e insuficiente para ser identificada; para el número restante (263 especies), logramos hacer una identificación confiable hasta especie de 242, a género de 19 y a familia de 2 . El descubrimiento de los textos ornitológicos y la existencia de ilustraciones originales ofrecen una vista única de la historia de la ornitología, así como de la historia del medio ambiente de México en general.

Manuscript received 8 May 2007; accepted 5 September 2007.

${ }^{5}$ E-mail: fcvg01@servidor.unam.mx 


\section{INTRODUCTION}

The rise of Mexican ornithology can be traced to its beginnings in the $1820 \mathrm{~s}$, with the explorations of Alexander von Humboldt, William Bullock, and Ferdinand Deppe (Stresseman 1954, Schifter 1996) and descriptions of new bird taxa by Charles Lucien Bonaparte and William Swainson (Swainson 1827, Mearns and Mearns 1998), among others. In the middle of the $19^{\text {th }}$ century, Phillip Sclater, Richard Sharpe, Tomasso Salvadori, and other ornithologists chiefly associated with the British Museum initiated a period of rapid growth in knowledge of Mexican birds, based on specimens collected by François Sumichrast, Auguste Sallé, Federico Craveri, Adolphe Boucard, and Matteo Botteri, among others (Binford 1989, Navarro 1989). This growth continued via the research of Osbert Salvin and Frederic DuCane Godman (Salvin and Godman 1879-1904) and by the Americans Edward Nelson and Edward A. Goldman (Goldman 1951). Nonetheless, the absence of Mexican ornithologists from the first half of the $19^{\text {th }}$ century is striking. Indeed, it was not until the 1860s that the Mexican ornithologists Rafael Montes de Oca, Manuel Villada, and Alfonso L. Herrera began to add to the knowledge of the birds of Mexico (Escalante et al. 1993).

The period preceding the known rise of Mexican ornithology, however, has long been associated with a gap in knowledge. Specifically, in contrast with other taxonomic groups (Sessé and Mociño 1893, 1894, McCoy and Flores-Villela 1985, Lamas 1986), the ornithological results of the Real Expedición Botánica a Nueva España (Royal Botanical Expedition to New Spain) have long been considered lost. This expedition was commanded by Martín de Sessé and was carried out between 1787 and 1803, covering New Spain (roughly, the territory of modern Mexico and the western United States), Guatemala, and areas north to Vancouver Island (McVaugh 1977; Fig. 1). The Mexican scientist José Mariano Mociño, born in Temascaltepec, Estado de México, joined the group as of 1790. This expedition was carried out contemporaneously with that of Alejandro Malaspina (González-Claverán 1988), which covered all the overseas possessions of Spain. The Sessé-Mociño expedition began 16 years prior to Alexander von Humboldt's arrival to
Mexico in 1803, just a few days after the return of Sessé and Mociño to Spain (Zamudio and Butanda 1999) following the conclusion of their expedition. This expedition occurred almost 100 years before the flourishing of Mexican ornithology referred to above.

It is known that the Real Expedición Botánica assembled a large amount of information about Mexican birds (Bernabéu-Albert et al. 2000). This information took the form of taxonomic descriptions, collected specimens, and paintings of many species. The latter were prepared by expedition artists Atanasio Echeverría and Vicente de la Cerda. However, the ornithological results of the Sessé-Mociño expedition have essentially been lost to science for more than the past 200 years (Gómez-Vázquez 1997, PuigSamper and Zamudio 1998).

The Sessé-Mociño ornithological results began to reappear when a small collection of original paintings, apparently from the expedition, was found. The paintings were identified in Barcelona in 1979 and purchased by the Hunt Institute for Botanical Documentation, Carnegie-Mellon University, Pittsburgh, in 1981 (Long-lost 1981, White et al. 1998). In 1997, Puig-Samper and Zamudio (1998) discovered unpublished manuscripts in the library of the Museo Nacional de Ciencias Naturales in Madrid that included descriptions of many bird species, again apparently from the Sessé-Mociño material. These discoveries, which represent an important portion of the overall ornithological results of the expedition, together make possible a broader study of the SesséMociño ornithology than with either source alone: a taxonomic list using modern nomenclature, analysis of correspondence between the paintings and the manuscripts, and conclusions regarding the provenance of this material.

\section{METHODS}

We had available 83 electronic images of the paintings housed in the Hunt Institute (White et al. 1998; Fig. 2A); we also used images of 23 additional paintings published by Arias Divito (1968) and previously identified by BarreiroRodríguez (1987). Finally, we had photocopies of the two manuscript volumes that had been found in the Museo Nacional de Ciencias Naturales in Madrid (Puig-Samper and Zamudio 1998; Fig. 2B). Both paintings and text had species names assigned, but the nomenclature 


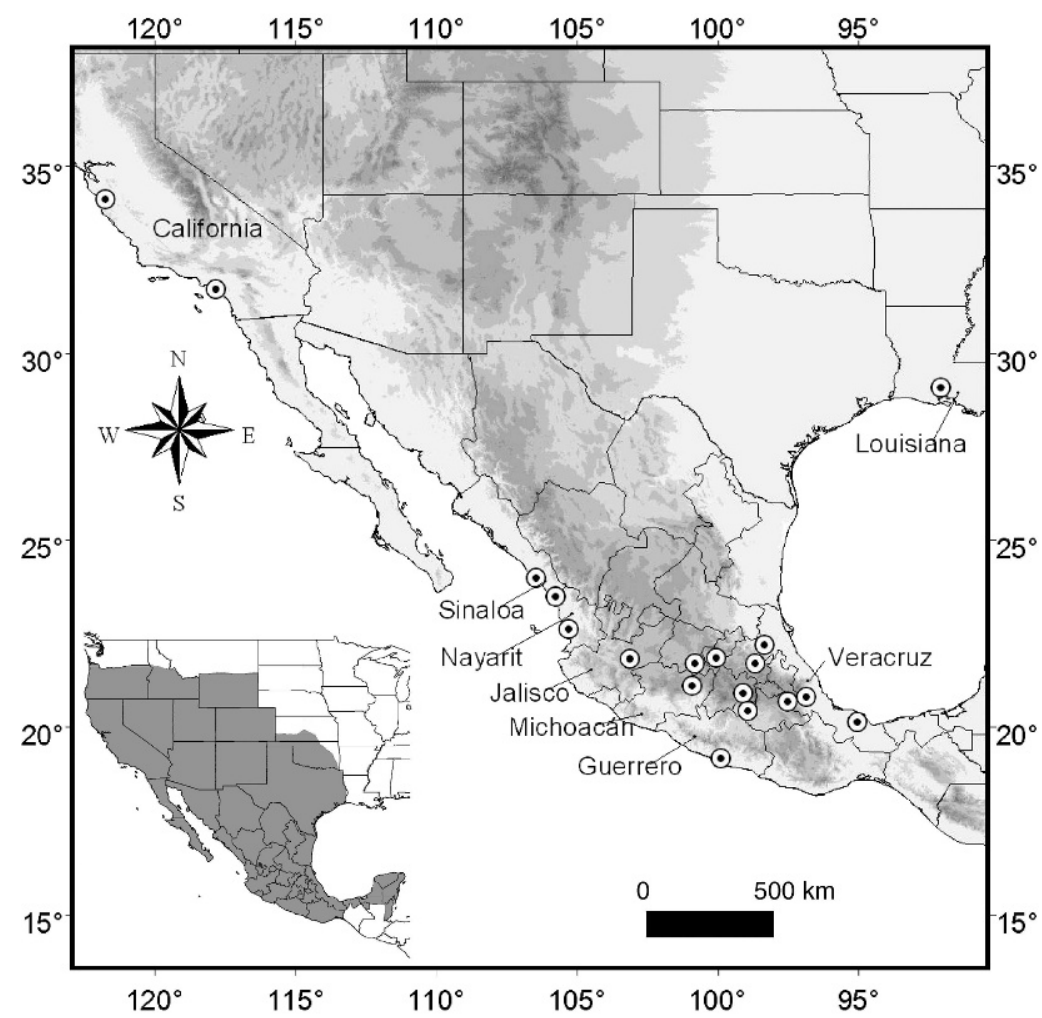

FIGURE 1. Map depicting geographic coverage of Mexican and U.S. localities in the text of the expedition manuscripts mentioned as collecting sites for the Sessé-Mociño expedition (dotted circles). Shading is based on the Digital Elevation Model of Hydro1K (U.S. Geological Survey 2006). Shaded portion of inset map depicts the approximate territorial coverage of Nueva España in the late eighteen century (Camelo et al. 2007).

used was so dated that the correspondence with more modern taxonomy (Dickinson 2003) was almost nil.

To assign names to the birds depicted in the paintings, AGN-S and ATP independently identified each to species (whenever possible) based on characteristics of plumage, size, and shape, with the help of field guides (Howell and Webb 1995, Sibley 2000) and occasional confirmation via inspection of specimens in the Museo de Zoología, Facultad de Ciencias, Universidad Nacional Autónoma de México. If the two identifications matched, we considered the identification to be correct; if they disagreed, however, we resolved the difference via detailed inspection and discussion. To identify taxa mentioned in the manuscripts, which were entirely in Latin, the same two authors reviewed the text with the help and support of specialized literature (Salvin and
Godman 1879-1904, Ridgway and Friedmann 1901-1950, Phillips 1986). The resulting lists were compared to nomenclature on the paintings and in the text, geographic records of species treated, and the temporal sequence of discovery and documentation of the Mexican avifauna.

\section{RESULTS}

Of a total of 83 paintings from the Hunt Institute that were available, we were able to identify 78 to species and 5 only to family. In the text, 290 species were treated, but for 27 , the descriptions were fragmentary and insufficient for identification (Table 1); of the remaining 263 species, we arrived at a species-level identification for 242 (of which one is identified only questionably, and two to the species complex only) and identified the remainder to genus (19) or family (two). Following is 

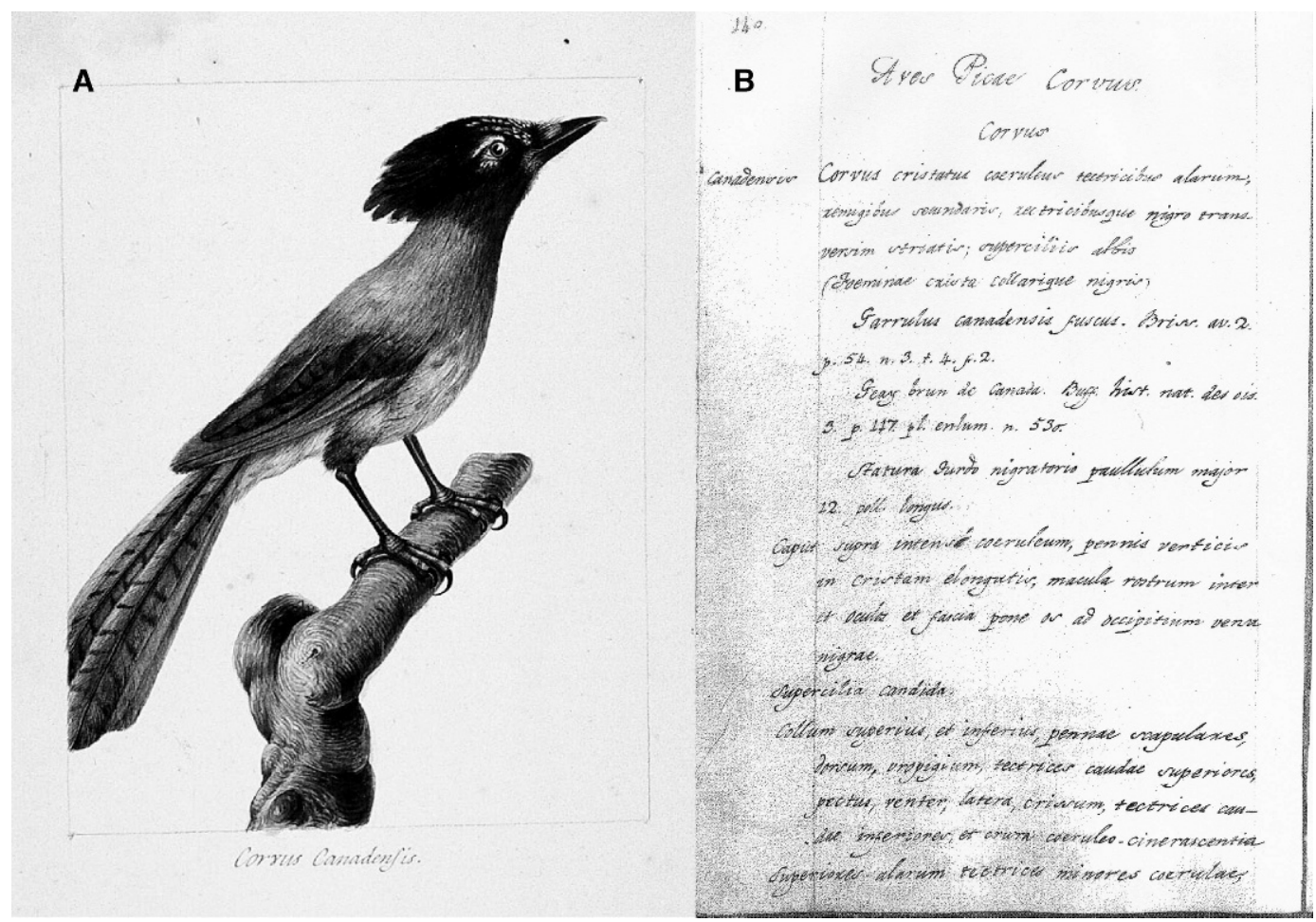

FIGURE 2. Examples of expedition materials used for analysis and identification of taxa. A) Sample of the original paintings from the expedition (Corvus Canadensis, presently Cyanocitta stelleri; White et al. 1998), courtesy of Hunt Institute for Botanical Documentation, Carnegie Mellon University, Pittsburgh, PA. B) Sample page of the species account of $C$. canadensis in the manuscripts housed at the Museo de Ciencias Naturales, Madrid.

a phylogentically ordered, annotated list of the taxa, (AOU 1998; with the exception of Cacatua moluccensis that was added at the end of Psittacidae) including the following information as available or discernable, formatted as shown: original name, [modern name, taxonomic authority and year of description of the modern taxon]; volume: page in the SesséMociño manuscripts, [correspondence with a painting if one exists $(\mathrm{H}=$ Hunt Institute, $\mathrm{MNCN}=$ Museo Nacional de Ciencias Naturales)]. Age and sex. Distribution and notes translated directly as stated in the manuscripts. (Our comments). Throughout, information that we added is placed in brackets. Question marks are added where information provided remains ambiguous. The Spanish word monte is translated as indicating undeveloped or wild areas (a very common colloquial expression in Mexican Spanish), as opposed to mountains as might be supposed.

\section{SPECIES LIST}

\section{ANATIDAE}

Anas dominica, [Dendrocygna autumnalis (Linnaeus 1758)]; 1:327. Tropical Mexico, such as Paramita [Nayarit].

Anas dimidiata, [Dendrocygna autumnalis (Linnaeus 1758)]; 1:334, [H]. Many sites across Mexico. (The text mentions "domesticated," but without explanation).

Anas anser, [Anser sp.]; 1:332. Europe and Mexico. Anas moschata, [Cairina moschata (Linnaeus 1758)]; 1:333, [H]. Domestic. Many sites across Mexico.

Anas strepera, [Anas strepera (Linnaeus 1758)]; 1:336, [H]. Lake Texcoco [Estado de México].

Anas variegata, [Anas discors (Linnaeus 1766)]; 1:342, [H]. Winters on Lake Texcoco [Estado de México].

Anas mexicana, [Anas clypeata (Linnaeus 1758)]; 1:337, [A painting $(\mathrm{H})$ carries this name, but the subject is identifiable only as Anas sp.]. Lake Texcoco [Estado de México].

Anas smaradigna, [Anas crecca (Linnaeus 1758)]; 1:346. Lake Texcoco [Estado de México]. 
TABLE 1. Species mentioned in expedition manuscripts for which identification was not possible because of overly brief or lacking species accounts in the text and lack of related paintings for comparison.

\begin{tabular}{|c|c|c|}
\hline Species & Volume & Page \\
\hline Ardea melanocephala & 1 & 432 \\
\hline Falco himantopus & 1 & 42 \\
\hline Falco melanetos & 1 & 18 \\
\hline Falco rufipes & 1 & 48 \\
\hline Falco undulatus & 1 & 46 \\
\hline Fringilla bicolor $^{\mathrm{a}}$ & 2 & 153 \\
\hline Fringilla carduelis & 2 & 169 \\
\hline Fringilla virescens ${ }^{\mathrm{b}}$ & 2 & 159 \\
\hline Hirundo americana & 2 & 302 \\
\hline Hirundo purpurea & 2 & 306 \\
\hline Hirundo rustica & 2 & 300 \\
\hline Hirundo urbica & 2 & 304 \\
\hline Motacilla fasciata & 2 & 265 \\
\hline Motacilla ferruginea & 2 & 257 \\
\hline Motacilla flaviventris & 2 & 261 \\
\hline Muscicapa arundinacea & 2 & 190 \\
\hline Muscicapa eremita & 2 & 188 \\
\hline Muscicapa fuliginosa & 2 & 225 \\
\hline Muscicapa minuta & 2 & 207 \\
\hline Muscicapa mississipensis & 2 & 201 \\
\hline Oriolus olivaceus & 1 & 181 \\
\hline Picus capistratus & 1 & 250 \\
\hline Rallus variegatus & 1 & 510 \\
\hline Scolopax nigri-rostris & 1 & 455 \\
\hline Tanagra ochracea & 2 & 147 \\
\hline Tanagra variabilis & 2 & 137 \\
\hline Turdus plumbipedis & 2 & 75 \\
\hline
\end{tabular}

${ }^{\text {a }}$ Locality: Acaponeta [Nayarit].

b Painting from Museo Nacional de Ciencias Naturales might represent Melospiza melodia or Passerella iliaca, but the depiction of the tail suggests that the depicted bird was molting, so it is possible that it was a juvenile or subadult.

Anas sima, [Anas sp.?]; 1:340. Lake Texcoco [Estado de México]. ico.

Anas albi-frons, [Anas sp.?]; 1:349. Tropical Mex-

Anas naevia, [Anas sp.?]; 1:351. Mexico.

Anas fusca, [Melanitta fusca (Linnaeus 1758)]; 1:330.

Mergus mexicanus, [Anatidae sp.]; 1:356. Lake Texcoco [Estado de México]. (The description in the text of a serrated bill suggests Mergus).

\section{CRACIDAE}

Phasianus motmot, [Ortalis vetula (Wagler 1830)]; 1:524. Tropical Mexico.

Phasianus viridescens, [Penelope purpurascens (Wagler 1830)]; 1:528, [H]. Tropical areas near Acayucan [Veracruz].

\section{PHASIANIDAE}

Phasianus gallus, [Gallus gallus (Linnaeus 1758)]; 1:522. Worldwide and in Mexico.

Meleagris gallapavo, [Meleagris gallopavo (Linnaeus 1758)]; 1:514. Europe and throughout Mexico.
(The former clearly refers to domestic individuals that had already been exported to Europe).

\section{ODONTOPHORIDAE}

Tetrao striatus, [Callipepla squamata (Vigors 1830)]; 2:12. Mexico.

Tetrao plumbeus, [Callipepla douglasii (Vigors 1829)]; 2:9.

Tetrao californicus, [Callipepla californica (Shaw 1798)]; 2:4, [MNCN]. Monterey [California, United States].

Tetrao ciliaris, [Colinus virginianus (Linnaeus 1758)]; 2:15. Female.

Tetrao guttatus, [Colinus virginianus (Linnaeus 1758)]; 2:7. Female.

Tetrao maculatus, [Cyrtonyx montezumae (Vigors 1830)]; $2: 1$.

PODICIPEDIDAE

Colymbus dominicus, [Tachybaptus dominicus (Linnaeus 1766)]; 1:377. Lake Texcoco [Estado de México] and the island of Santo Domingo.

PHALACROCORACIDAE

Pelecanus carbo, [Phalacrocorax auritus? (Lesson 1831)]; 1:369. Coastal areas of the oceans of Asia; near Acapulco.

ANHINGIDAE

Plotus anhinga, [Anhinga anhinga (Linnaeus 1766)]; 1:371. Female or immature. Lake Texcoco [Estado de México].

FREGATIDAE

Pelecanus leucocephalus, [Fregata magnificens (Mathews 1914)]; 1:367. Immature. Gulf of Mexico. Pelecanus aquilus, [Fregata magnificens (Mathews 1914)], 1:362. Adult male. Gulf of Mexico.

\section{ARDEIDAE}

Ardea serratirrostris, [Botaurus lentiginosus (Rackett 1813)]; 1:415, [MNCN]. (The painting for this species is labeled also as Ardea-ciconia Mexicana).

Ardea minuta, [Ixobrychus exilis (Gmelin 1789)];

1:428, [H]. Lake Texcoco [Estado de México].

Ardea striata, [Tigrisoma mexicanum (Swainson 1834)]; 1:417, [H]. Immature? Rivers near Apatzingán [Michoacán].

Ardea hudsonias, [Ardea herodias (Linnaeus 1758)];

1:409. Rivers near Amoles [Querétaro?]. Ardea alva, [Ardea alba (Linnaeus 1758)]; 1:414. In many Mexican lakes.

Ardea garceta, [Egretta thula (Molina 1782)]; 1:427. In Mexican lakes and coasts of the oceans of Asia.

Ardea virescens, [Butorides virescens (Linnaeus 1758)]; 1:422. Mexico, the Carolinas, Virginia, Martinica, and Cayenne.

Ardea nycticorax, [Nycticorax nycticorax (Linnaeus 1758)]; 1:438, [H]. Lake Texcoco [Estado de México].

Ardea naevia, [Nycticorax nycticorax (Linnaeus 1758)]; 1:430. Immature. Lake Tixtla [Guerrero]. Ardea cayenensis, [Nyctanassa violacea (Linnaeus 1758)]; 1:419, [H]. Tropical parts of Mexico.

Ardea castanea, [Cochlearius cochlearius (Linnaeus 1766)]; 1:436. Immature. Lake Texcoco [Estado de México].

Ardea crasi-rostris, [Cochlearius cochlearius (Linnaeus 1766)]; 1:434. Immature. Lake Tixtla [Guerrero]. 
Cancroma coclearia, [Cochlearius cochlearius (Linnaeus 1766)]; 1:401. Rivers of Mexico and Cayenne. THRESKIORNITHIDAE

Platalea ajaja, [Platalea ajaja (Linnaeus 1758)]; 1:395. The Brazilian Guianas and the lakes of Mexico.

\section{CICONIIDAE}

Ardea emarginata, [Mycteria americana (Linnaeus 1758)]; 1:407, [H]. Lakes near Tepic [Nayarit].

CATHARTIDAE

Vultur aura, [Cathartes aura (Linnaeus 1758)]; 1:3. Vultur papa, [Sarcoramphus papa (Linnaeus 1758)]; 1:1. Mexico.

\section{ACCIPITRIDAE}

Falco coronatus, [Pandion haliaetus (Linnaeus 1758)]; 1:27. Tropical fields near San Blas [Nayarit].

Falco cinereus, [Circus cyaneus (Linnaeus 1766)]; 1:33. Tropical Mexico.

Falco naevius, [Accipiter cooperii (Bonaparte 1828)]; 1:44, [H]. Temperate mountains between

San Antonio and San Felipe del Obraje [Valle de Toluca, Estado de México].

Falco fuscus, [Buteogallus anthracinus (Deppe

1830)]; 1:5, [H]. ("Falco communis" is crossed out.)

Falco gemebundus, [Parabuteo unicinctus (Temminck 1824)]; 1:40. Sinaloa.

Falco albi-ventris, [Buteo albicaudatus (Vieillot 1816)]; 1:29, [H]. Throughout Mexico.

Falco butteo, [Buteo jamaicensis (Gmelin 1788)]; 1:21, $[\mathrm{H}]$. Mexico.

Falco crassirostris, [Buteo sp.]; 1:23. Mexico.

Falco arpyja, [Harpia harpyja (Linnaeus 1758)]; 1:16. Mexico. (The text describes a Harpy Eagle clearly and unequivocally, but the painting carrying this name is of Caracara cheriway [see below]).

\section{FALCONIDAE}

Bultur harpyja, [Caracara cheriway (Jacquin 1784)]. [Image only (MNCN)].

Falco rufi-ventris, [Herpetotheres cachinnans (Linnaeus 1758$)] ; 1: 50,[\mathrm{H}]$. Mexico.

Falco cyanopterus, [Falco sparverius (Linnaeus 1758)]; 1:31, [H]. Mexico City area.

Falco noveboracensis, [Falco sparverius (Linnaeus 1758)]; 1:19. Female. Mexico.

Falco sparverius, [Falco sparverius (Linnaeus 1758)]; 1:13, [H]. Male. Mexico.

Falco lithofalco, [Falco columbarius (Linnaeus 1758)]; 1:11, [H]. Mexico.

Falco albi-frons, [Falco femoralis? (Temminck 1822)]; 1:38, [H]. Mountains near Mexico City.

Falco ciliaris, [Falco mexicanus (Schlegel 1851)]; 1:35, $[\mathrm{H}]$. Mexico.

\section{RALLIDAE}

Tringa pusilla (Scolopax rufa), [Rallus limicola (Vieillot 1819)]. [Image only (MNCN)].

Rallus olivaceus, [Aramides cajanea (Müller 1776)];

1:508, [H]. Tropical Acayucan [Veracruz].

Fulica mexicana, [Porzana carolina (Linnaeus 1758)]; 1:491. Lake Texcoco [Estado de México].

Rallus undatus, [Porzana carolina (Linnaeus 1758)]; 1:506, [MNCN]. Lake Texcoco [Estado de México].

Fulica chloropus, [Gallinula chloropus (Linnaeus 1758)]; 1:495, [MNCN]. Canals of Mexico City.

\section{GRUIDAE}

Ardea mexicana, [Grus canadensis (Linnaeus 1758)]; 1:412, [H]. Lakes across Mexico. (The painting is of Nycticorax nycticorax, but the description is of Grus canadensis).

\section{CHARADRIIDAE}

Tringa squatarola, [Pluvialis squatarola (Linnaeus 1758)]; 1:462. Rivers and lakes of Mexico and Europe.

Charadrius torquatus, [Pluvialis sp.]; 1:473.

Charadrius albifrons, [Charadrius semipalmatus (Bonaparte 1825)]; 1:478. Lakes of Mexico. Charadrius vociferus, [Charadrius vociferus (Linnaeus 1758)]; 1:475. Mexico.

Charadrius aegyptius, [Charadrius sp.]; 1:471.

RECURVIROSTRIDAE

Charadrius himantopus, [Himantopus mexicanus (Müller 1776)]; 1:472, [H, MNCN]. Coastal areas of the Asian oceans, close to Coahuayana [Michoacán].

Recurvirostris avocetta, [Recurvirostra americana (Gmelin 1789)]; 1:485, [MNCN]. Lakes of Mexico and southern Europe.

JACANIDAE

Parra mexicana, [Jacana spinosa (Linnaeus 1758)]; 1:501.

SCOLOPACIDAE

Tringa guttata, [Actitis macularius (Linnaeus 1766)]; 1:458. Tropical Mexico.

Tringa ochropus, [Tringa semipalmata (Gmelin 1789)]; 1:464.

Scolopax superciliaris, [Tringa $\mathrm{sp}$.]; 1:451.

Tringa ganga, [Bartramia longicauda (Bechstein 1812)]; 1:466. Mexican grasslands.

Tringa minuta, [Calidris sp.]; 1:461. Tropical swamps [marshes?] of Mexico.

Scolopax gallinago, [Gallinago delicata (Ord 1825)];

1:447. Lake Texcoco [Estado de México].

Scolopax longipeda, [Limnodromus sp.]; 1:452.

LARIDAE

Larus ichthyaetus, [Larus atricilla (Linnaeus 1758)]; 1:387. Lake Texcoco [Estado de México].

Larus naevius, [Larus sp.]; 1:385.

\section{ALCIDAE}

Diomedea exulans, [Brachyramphus marmoratus (Gmelin 1789)]. [Image only (MNCN)]. (This image, erroneously labeled as $D$. exulans, likely pertains to material obtained on the expedition to Nootka, although there are occasional Mexican records [particularly off the north coast of Baja California, AOU 1998]).

Alca cirrhata, [Fratercula cirrhata (Pallas 1769)]; 1:359. Open ocean between Kamchatka and Nootka Island [Alaska, United States].

COLUMBIDAE

Columba hispanica, [Columba livia (Gmelin 1789)] 2:23. Mexico City and Europe.

Columba domestica, [Columba livia (Gmelin 1789)]; 2:21. Mexico and Europe.

Columba turtur hybridus, [Patagioenas flavirostris (Wagler 1831)]; 2:25. Mexico and Europe. (Although the textual description led us to conclude $P$. flavirostris, we do not discard the possibility of some odd domestic form or a hybrid between Columba and Streptopelia that may have been brought to Mexico 
by the Europeans, although we know of no documentation of such early introductions).

Columba ferox, [Patagioenas fasciata (Say 1823)]; 2:30, $[\mathrm{H}]$. Mountains near San Nicolás [Distrito Federal].

Columba leucoptera, [Zenaida asiatica (Linnaeus 1758)]; 2:28. Mexico and Asia.

Columba rufi-ventris, [Zenaida macroura (Linnaeus 1758)]; 2:32, [H]. Mexico.

Columba marginata, [Columbina inca (Lesson 1847)]; 2:27.

Columba longicauda, [Columbina passerina (Linnaeus 1758)]; 2:39.

Columba cinerea, [Claravis pretiosa (Ferrari-Pérez 1886)]; 2:35, [H].

Columba coerulea (corrected to C. cinerea), [Claravis pretiosa (Ferrari-Pérez 1886)]; 2:37, [H]. Los Tuxtlas [Veracruz].

\section{PSITTACIDAE}

Psittacus canicularis, [Aratinga canicularis (Linnaeus 1758)]; 1:100. Tropical Mexico. (Although the textual description omits mention of the orange forehead of this species [at least in adults], the remainder of the text fits well. This species is the only member of the genus Aratinga present in the lowlands of western Mexico).

Psittacus militaris, [Ara militaris (Linnaeus 1766)]; 1:94. Tropical Mexico.

Psittacus armillaris, [Rhynchopsitta pachyrhyncha (Swainson 1827)]; 1:104. Tropical areas of Mexico.

Psittacus brevicaudus, [Bolborhynchus lineola (Cassin 1853)]; 1:98. Tropical areas near Huauchinango [Puebla] and in the Huasteca [northeastern Mexico].

Psittacus leucocephalus, [Amazona albifrons (Sparrman 1824)]; 1:107. Acaponeta [Nayarit].

Psittacus festivus, [Amazona finschi (Sclater 1864)];

1:96. Tropical areas near Acaponeta [Nayarit].

Psittacus autumnalis, [Amazona autumnalis (Linnaeus 1758)]; 1:92. Tropical Mexico.

Psittacus ochropterus, [Amazona oratrix (Ridgway 1887)]; 1:105. Mexico.

Psittacus molucensis, [Cacatua moluccensis, (Gmelin 1788)]; 1:102. Jolo Island [southern Philippines], from where it was transported to Mexico.

CUCULIDAE

Cuculus americanus, [Coccyzus americanus (Linnaeus, 1758)]; 1:217, [H]. Temperate montane areas of Mexico.

Cuculus cayanus, [Piaya cayana (Linnaeus 1766)]; 1:219. Temperate mountain areas near Uruapan [Michoacán]. (Given plumage descriptions, refers to the form P. c. mexicana of western Mexico).

Phasianus parvulus, [Morococcyx erythropygus (Lesson 1842)]; 1:527. San Blas [Nayarit] near the ocean.

Phasianus mexicanus, [Geococcyx velox (Wagner 1836)]; 1:518.

Phasianus cursor, [Geococcyx californianus (Lesson 1829)]. [Image only (MNCN)].

Crotophaga ani, [Crotophaga sulcirostris (Swainson 1827)]; 1:130, [MNCN, H]. Africa and America.

STRIGIDAE

Strix bubo, [Bubo virginianus (Gmelin 1788)]; 1:69. CAPRIMULGIDAE

Caprimulgus mexicanus, [Chordeiles sp.]; 2:316.
Caprimulgus americanus, [Chordeiles sp.]; 2:313. Mexico.

Caprimulgus semitorquatus, [Caprimulgus sp.]; 2:318, [H]. Tropical areas of Mexico and Cayenne.

TROCHILIDAE

Trochilus macrourus, [Chlorostilbon canivetii (Lesson 1832)]; 1:306. Temperate areas of Mexico City, Mexico in general, and Cayenne. (Identifiable only as part of the $C$. canivetii complex).

Trochilus minutus, [Cynanthus latirostris (Swainson 1827)]; 1:295. Immature. Orchards near Guadalajara [Jalisco].

Trochilus septem-color, [Hylocharis leucotis (Vieillot 1818)]; 1:304. Temperate areas near Mexico City, Mexico in general, and Cayenne.

Trochilus pertinax, [Amazilia beryllina (Lichtenstein 1830)]; 1:318. Fields near Toluca [Estado de México].

Trochilus mexicanus, [Amazilia beryllina (Lichtenstein 1830)]; 1:312. Orchards near San Angel [Distrito Federal].

Trochilus coronatus, [Amazilia violiceps or A. cyanocephala (Gould 1859)]; 1:300. Temperate areas of Mexico City, Mexico in general, and Cayenne.

Trochilus colubris, [Lampornis amethystinus (Swainson 1827)]; 1:310. Orchards near San Angel [Distrito Federal].

Trochilus turpis, [Lampornis clemenciae (Lesson 1829)]; 1:308 [MNCN; shows both male and female]. Near Mexico City, and in temperate areas across Mexico.

Trochilus albi-ventris, [Calothorax lucifer (Swainson 1827)]; 1:316. Female. Fields near Tulancingo [Hidalgo].

Trochilus pulcherrimus, [Calothorax lucifer (Swainson 1827)]; 1:314. Male. Orchards near San Angel. [Distrito Federal].

Trochilus mosquitus, [Calypte costae? (Bourcier 1839)]; 1:320.

Trochilus flameus, [Selasphorus rufus (Gmelin 1788)]; 1:302. Temperate areas of Mexico City, Mexico in general, and Cayenne.

Trochilus pegasus, Trochilidae sp.];1:297. Female. Temperate areas of Mexico City, Mexico in general, and Cayenne.

TROGONIDAE

Trogon flavi-ventris, [Trogon melanocephalus (Gould 1835)]; 1:206.

Trogon viridis, [Trogon citreolus (Gould 1835)]; 1:203. Tropical Mexico, Brazil, and Peru.

Trogon strigilatus, [Trogon violaceus (Gmelin 1788)]; 1:205.

Trogon curucui, [Trogon mexicanus (Swainson 1827)]; 1:199. Montane areas near Malacatepec [Estado de México]. (Female described separately).

Trogon rufus, [Trogon elegans (Gould 1834)]; 1:207.

MOMOTIDAE

Ramphastos gressorius, [Momotus mexicanus (Swainson 1827)]; 1:118. Mexico.

Ramphastos motmota, [Momotus momota (Linnaeus 1766)]; 1:123.

ALCEDINIDAE

Alcedo alcyon, [Megaceryle alcyon (Linnaeus 1758)]; 1:261, [H], Female or immature. River margins across Mexico. 
Alcedo torquata, [Megaceryle alcyon (Linnaeus 1758)]; 1:264, [H; appears to refer to $M$. torquata, but description definitely is of $M$. alcyon], Male. Mexico.

Alcedo americana, [Chloroceryle americana (Gmelin 1788)]; 1:257, [H]. River edges across Mexico. (See notes under $A$. mexicana).

Alcedo mexicana, [Chloroceryle americana (Gmelin 1788)]; 1:259, [H]. Along the margins of the aqueduct of Querétaro. (These descriptions are somewhat problematic, as the male is described as A. americana, but a male and a female are described as this species. This multiple description may have been accidental or may have been based on some minor difference among specimens available).

\section{RAMPHASTIDAE}

Ramphastos mexicanus, [Aulacorhynchus prasinus (Gould 1834)]; 1:122. Tropical areas close to the Gulf Coastal lowlands.

Ramphastos aracari, [Pteroglossus torquatus (Gmelin 1788)]; 1:125. Tropical areas of Mexico.

Ramphastos picatus, [Ramphastos sulfuratus (Lesson 1830)]; 1:120. Tropical zone near Huehuetla [Puebla].

PICIDAE

Picus occidentalis, [Melanerpes formicivorus (Swainson 1827)]; 1:234. Mountains near Temascaltepec [Estado de México]. (The description is of an individual of the form $M$. $f$. formicivorus of central Mexico).

Picus maculatus, [Melanerpes pucherani (Malherbe 1849)]; 1:240. Tropical Mexico.

Picus mexicanus, [Melanerpes chrysogenys (Vigors 1839)]; 1:243. Tropical and temperate regions of Mexico. (Notes in the text indicate that at first this form was considered to refer to $M$. pucherani, but careful observation led them to conclude that it was a [new] species distinct from $M$. pucherani).

Picus murinus, [Picoides scalaris (Wagler 1829)]; $1: 248$.

Picus villosus, [Picoides villosus (Linnaeus 1766)]; 1:246. The Carolinas, Virginia, and Mexico. (The description in the text is of a member of the form $P$. $v$. jardinii, which is found in central and southern Mexico, although the distributional information provided is broader).

Picus molucensis, [Picoides stricklandi (Malherbe 1845)]; 1:236. Mountains around the valley close to Temascaltepec [Estado de México].

Picus rufescens, [Veniliornis fumigatus (D’Orbigny 1840)]; 1:251.

Picus lacertinus, [Piculus rubiginosus (Swainson 1820)]; 1:238. Tropical Mexico.

Yunx americanus, [Colaptes auratus (Linnaeus 1758)]; 1:227. Temperate mountains of Mexico. (The description refers to the form C. a. cafer).

\section{FURNARIIDAE}

Certhia picacea, [Lepidocolaptes leucogaster (Swainson 1827)]; 1:288, [H].

\section{TYRANNIDAE}

Muscicapa sp., [Sayornis nigricans (Swainson 1827)]; 2:212, [MNCN; labeled Tanagra dominica as well as Muscicapa nigra "Sp. N." with slightly different type]. Tropical regions of Mexico.
Muscicapa grisola, [Pyrocephalus rubinus (Boddaert 1783)]; 2:216. Female. Europe and Mexico. (No explanation given or available for mention of occurrence in Europe).

Lanius madagascarensis, [Myiarchus sp.]; 1:79. Tropical fields near Cuernavaca [Morelos].

Lanius sulphuratus, [Pitangus sulphuratus (Linnaeus 1766)]; 1:82. Mexico. (Interestingly, the female is described separately and incorrectly-the female described is likely a Tyrannus).

Muscicapa grandi-rostris, [Pitangus sulphuratus (Linnaeus 1766)]; 2:221.

Muscicapa cayanensis, [Myiozetetes similis (Spix 1825)]; 2:209, [MNCN]. Tropical Mexico.

Muscicapa longicauda, [Tyrannus forficatus (Gmelin 1789)]; 2:218. Fields near Zacatlán de las Manzanas [Puebla].

Muscicapa forficata, [Tyrannus savana (Vieillot 1808)]; 2:223.

Lanius nigrifrons, [Tityra semifasciata (Spix 1825)]; 1:81. Tropical Mexico.

LANIIDAE

Lanius excubitor, [Lanius ludovicianus (Linnaeus 1766)]; 1:77. Mountains near the Valle de México and in Louisiana.

VIREONIDAE

Tanagra crassirostris, [Cyclarhis gujanensis (Gmelin 1789)]; 2:142. Mexico.

CORVIDAE

Corvus stelleri, [Cyanocitta stelleri (Gmelin 1788)]; $1: 145,[\mathrm{H}]$. Mountains near Mexico City.

Corvus canadensis, [Cyanocitta stelleri (Gmelin 1788)]; $1: 140,[\mathrm{H}]$. Temperate montane areas of Mexico. (Description refers to the form C. s. coronata).

Coracias torquata, [Calocitta formosa (Swainson 1827)]; 1:160, [H]. Tropical Mexico.

Corvus cyanocephalus, [Cyanocorax yncas (Boddaert 1783)]; 1:136, [H]. Tropical regions of the Huasteca [northeastern Mexico]. (The authors compare this form with that of Peru, and note phenotypic differences).

Corvus fuscus, [Cyanocorax morio (Wagler 1829)]; 1:149. Tropical Mexico. (Description refers to the form C. m. morio, of eastern Mexico).

Corvus melanocephalus, [Cyanocorax beecheii (Vigors 1829)]; 1:148. Immature? Shaded regions of Sinaloa. (Some aspects of the description suggest Cyanocorax sanblasianus, for which this record would be an extension of range and would probably be unlikely).

Corvus coronatus, [Cyanolyca cucullata (Ridgway 1885)]; 1:153, [H].

Corvus californicus, [Aphelocoma californica (Vigors 1839)]; 1:151, [H]. Upper California (modern United States).

Corvus bicolor, [Aphelocoma ultramarina (Bonaparte 1825)]; 1:138, [H]. Mountains of the Desierto de los Leones [Distrito Federal].

Corvus coeruleus, [Aphelocoma unicolor (Du Bus de Gisignies 1847)]; 1:144, [H]. Mountains near Zacatlán [Puebla].

Corvus corone, [Corvus corax (Linnaeus 1758)]; 1:147. Europe and Mexico.

ALAUDIDAE

Alauda torquata, [Eremophila alpestris (Linnaeus 1758)]; 2:49, [H]. Fields in Puebla. 


\section{HIRUNDINIDAE}

Hirundo violacea, [Progne subis (Linnaeus 1758)]; 2:291:[H], Male. Louisiana. Rarely in Mexico.

Hirundo subis, [Progne chalybea (Gmelin 1789)]; 2:293, [H]. Tropical Mexico.

Hirundo vera-crucensis, [Tachycineta albilinea (Lawrence 1863)]; 2:289.

Hirundo mexicana, [Stelgidopteryx serripennis (Audubon 1838)]; 2:298, [H].

Hirundo tapera, [Stelgidopteryx serripennis (Audubon 1838)]; 2:295. Mexico, arriving in April.

PARIDAE

Parus melanocephalus, [Poecile sclateri (Kleinschmidt 1897)]; 2:279. Mountains of the Desierto de los Leones [Distrito Federal].

AEGITHALIDAE

Pipra minuta, [Psaltriparus minimus (Townsend 1837)]; 2:273. Mountains of the Desierto de los Leones [Distrito Federal]. (Refers to the form P. m. melanotis).

\section{CERTHIIDAE}

Certhia familiaris, [Certhia americana (Bonaparte 1838)]; 1:277. Mexico, Spain and Europe. (The painting labeled with this name is likely Troglodytes aedon, although the description fits well with Certhia americana).

\section{TROGLODYTIDAE}

Certhia rufiventris, [Campylorhynchus zonatus (Lesson 1832)]; 1:283. Tropical Mexico.

Turdus varius, [Campylorhynchus megalopterus (Lafresnaye 1845)]; 2:78. Desierto de los Leones [Distrito Federal].

Certhia naevia, [Campylorhynchus jocosus (Sclater 1859)]; 1:285. Tropical Mexico.

Certhia miniata, [Salpinctes obsoletus (Say 1823)]; 1:281, [H]. Mexico.

Certhia canora, [Catherpes mexicanus (Swainson 1829)]; 1:273, [H].

Certhia minuta, [Thryomanes bewickii (Audubon 1827)]; 1:275. Mountains near the Desierto de los Leones [Distrito Federal]. (The painting labeled with this name depicts Cyanerpes cyaneus, for which no description is available in the text, whereas the description likely is of Thryomanes bewickii).

REGULIDAE

Motacilla flavicapilla, [Regulus satrapa (Lichtenstein 1823)]; 2:269. Desierto de los Leones [Distrito Federal].

\section{SYLVIIDAE}

Motacilla vulgarissima, [Polioptila melanura (Lawrence 1857)]; 2:239. Temperate Mexico.

Motacilla bicolor, [Polioptila sp. (Sclater 1855)]; 2:237. Mexico. (If the individual described were a male, this description would be of $P$. caerulea).

Muscicapa marginata, [Polioptila sp.]; 2:199.

\section{TURDIDAE}

Muscicapa serrana, [Sialia sialis (Linnaeus 1758)];

2:197. Near Huasutepeque [Morelos].

Muscicapa ochracea, [Myadestes occidentalis (Stegnejer 1882)]; 2:204. Mexico.

Turdus flavi-rostris, [Catharus aurantiirostris (Hartlaub 1850)]; 2:65, [MNCN; labeled Turdus arundinaceus and Pipra cinerea in slightly different type]. Orchards of San Angel [Distrito Federal].
Turdus testaceus, [Turdus grayi (Bonaparte 1838)]; 2:69. Mexico.

Turdus migratorius, [Turdus migratorius (Linnaeus 1766)]; 2:63. Fields near Toluca [Estado de México]. MIMIDAE

Turdus melanocephalus, [Dumetella carolinensis (Linnaeus 1766)]; 2:77.

Turdus dominicus, [Mimus polyglottos (Linnaeus 1758)]; 2:71. Throughout Mexico.

Turdus musicus, [Toxostoma ocellatum (Sclater 1862)]; 2:67. Mexico.

Certhia sibilans, [Toxostoma curvirostre (Swainson 1827)]; 1:279, [MNCN; labeled Merops fuscus "Sp. N.']. Temperate Mexico.

Oriolus canorus, [Melanotis caerulescens (Swainson 1827)]; 1:179. Tropical Mexico.

Turdus coeruleus, [Melanotis caerulescens (Swainson 1827)]; 2:73. Near Mazatlán.

\section{PTILOGONATIDAE}

Muscicapa giratrix, [Ptilogonys cinereus Swainson

1824]; 2:202. Orchards close to Mexico City.

Muscicapa nitens, [Phainopepla nitens (Swainson 1837)]; 2:184. Mexico.

\section{PARULIDAE}

Motacilla maderaspatana, [Vermivora ruficapilla? (Wilson 1811)]; 2:248, [MNCN]. Near Mexico City. (Barreiro-Rodríguez [1987] identified this painting as referring to Vireo philadelphicus).

Motacilla aestiva, [Dendroica petechia (Linnaeus 1766)]; 2:253. Adult female. Orchards near San Agustín [Distrito Federal].

Motacilla bifasciata, [Dendroica dominica (Linnaeus 1766)]; 2:263.

Motacilla flavifrons, [Dendroica sp.]; 2:243. Temperate Mexico. (The description could refer to any of D. townsendi, D. occidentalis, or D. virens).

Motacilla mexicana, [Dendroica sp.]; 2:255. Fields of the Valle de México.

Motacilla varia, [Mniotilta varia (Linnaeus 1766)]; 2:235. Fields near Toluca [Estado de México].

Motacilla brevicauda, [Seiurus motacilla (Vieillot 1808)]; 2:267.

Motacilla tepicensis, [Geothlypis trichas (Linnaeus 1766)]; 2:250. Fields near Tepic [Nayarit]. (Description refers to the migratory form).

Motacilla nigrifrons, [Geothlypis trichas (Linnaeus 1766)]; 2:241. Near the Valle de México. (Description refers to the resident form, G. t. melanops).

Motacilla mitrata, [Wilsonia citrina (Boddaert 1783)]; 2:259.

Motacilla pileata, [Wilsonia pusilla (Wilson 1811)]; 2:252, [MNCN; labeled Motacilla lutea and Motacilla coronata in a slightly different type]. Fields near Tepic [Nayarit].

Muscicapa tanagra mississip (the text has the label "ferruginea" crossed out), [Ergaticus ruber (Swainson 1827)]; 2:183. Near the Desierto de los Leones [Distrito Federal].

Muscicapa sanguinea, [Ergaticus ruber (Swainson 1827)]; 2:186. Immature. Near the Desierto de los Leones [Distrito Federal].

Muscicapa tricolor, [Myioborus pictus (Swainson 1829)]; 2:192. Near Toluca [Estado de México].

Muscicapa cinerea, [Myioborus miniatus (Swainson 1827)]; 2:195. Fields near Toluca [Estado de México]. 
Muscicapa militaris, [Myioborus miniatus (Swainson 1827)]; 2:227. Desierto de los Leones [Distrito Federal].

Muscicapa virens, [Icteria virens (Linnaeus 1758)]; 2:214. The Carolinas and Mexico.

Motacilla longi rostris, [Granatellus venustus (Bonaparte 1850)]; 2:245. Near Huasatepeque [Morelos]. THRAUPIDAE

Tanagra rufa, [Habia sp.]; 2:146. Los Tuxtlas [Veracruz].

Tanagra mississipensis, [Piranga flava (Vieillot 1822)]; 2:139. Near the Valle de México.

Fringilla americana, [Piranga ludoviciana (Wilson 1811)]; 2:165, [H]. Near the Valle de México.

Tanagra olivacea, [Piranga sp.]; 2:129. Zitácuaro [Michoacán].

Tanagra cyanocephala, [Thraupis abbas (Deppe 1830)]; 2:140. Mexico.

\section{EMBERIZIDAE}

Loxia minima, [Sporophila torqueola (Bonaparte 1850)]; 2:113. (The description is of the western Mexican form, S. t. torqueola).

Loxia nigra, [Oryzoborus funereus (Sclater 1829) or Sporophila aurita (Bonaparte 1850)]; 2:114. Mexico.

Fringilla flavi-ventris, [Atlapetes pileatus? (Wagler 1831)]; 2:157, [H]. Mountains near the Valle de México.

Loxia americana, [Pipilo maculatus (Swainson 1827)]; 2:115, [MNCN]. San Angel [Distrito Federal]. (The description is of the form $P$. m. macronyx, of the Mexican Transvolcanic Belt).

Tanagra fusca, [Pipilo fuscus (Swainson 1827)]; 2:133. Mexico.

Tanagra trifasciata, [Aimophila ruficauda (Bonaparte 1853)]; 2:127. Temperate areas near Zitácuaro

[Michoacán].

Alauda trifasciata, [Aimophila ruficauda (Bonaparte 1853)]; 2:47.

Tanagra superciliaris, [Oriturus superciliosus (Swainson 1837)]; 2:131. Fields near Toluca [Estado de México].

Fringilla superciliaris, [Spizella passerina (Bechstein 1798)]; 2:167. Fields of the Valle de México.

Tanagra murina, [Spizella atrogularis (Cabanis 1851)]; 2:135. Fields near Toluca [Estado de México].

Fringilla fusco-ferruginea, [Passerculus or Melospiza]; 2:163. Fields near San Nicolás [Distrito Federal]. (This description could refer to various sparrow genera with streaked chests).

Fringilla albi-ventris, [Passerculus or Melospiza]; 2:173. Fields of the Valle de México. This description could refer to various sparrows genera with streaked chests.

Fringilla curbirostra, [Junco phaeonotus (Wagler 1831)]; 2:161, [H]. Fields of the Valle de México.

CARDINALIDAE

Colius torquatus, [Saltator atriceps (Lesson 1832)]; 2:87. Hueytlalpan [Puebla].

Tanagra gullaris, [Caryothraustes poliogaster $(\mathrm{Du}$ Bus de Gisignies 1847)]; 2:125. Los Tuxtlas [Veracruz].

Colius trucidatus, [Rhodothraupis celaeno (Deppe 1830)]; 2:90. Tropical Mexico.

Loxia cardinalis, [Cardinalis cardinalis (Linnaeus 1758)]; 2:109. Mexico.
Loxia flavicans, [Pheucticus chrysopeplus (Vigors 1832)]; 2:97. Mazatlán [Sinaloa].

Loxia testacea, [Pheucticus melanocephalus (Swainson 1827)]; 2:100. Mountains of the Desierto de los Leones [Distrito Federal].

Loxia cyanea, [Passerina caerulea (Linnaeus 1758)]; 2:111.

Fringilla nigro coerulea, [Passerina cyanea (Linnaeus 1766)]; 2:171, [H].

ICTERIDAE

Oriolus phoeniceus, [Agelaius phoeniceus (Linnaeus 1766)]; 1:167, [MNCN].

Sturnus ludovicianus, [Sturnella sp.]; 2:57. Mexico and Louisiana.

Oriolus icterocephalus, [Xanthocephalus xanthocephalus (Bonaparte 1826)]; 1:175, [MNCN, but not examined by Barreiro-Rodríguez (1987)]. Mexico and Cayenne.

Corvus senegalensis, [Quiscalus mexicanus (Gmelin 1788)]; 1:142. Military post in Paramita [Nayarit]. (This species appears to have been identified as $C$. senegalensis based on the description of Linnaeus [1766], which pointed out the long, cupped tail).

Gracula quiscula, [Quiscalus mexicanus (Gmelin 1788)]; 1:195, [MNCN]. Mexico.

Apelis continga, [Quiscalus palustris (Swainson 1827)]. [MNCN; show both male and female]. (These paintings clearly refer to this species [BarreiroRodríguez 1987], which is extinct but was endemic to the the lakes of the Valle de México and neighboring marsh areas [Peterson 1998]. Although both plates had the name Gracula quiscula added below [therefore referring to $Q$. mexicanus, we can confirm the identification as this species based on diagnostic characters shown in the paintings [bill shape and female coloration]. Nonetheless, no description in the text allows differentiation from $Q$. mexicanus, so examination of the specimens may not have led Mociño to see them as two species. These images may be the only ones of this species made directly from fresh material).

Oriolus auratus, [Molothrus aeneus (Wagler 1829)]; 1:183. Tropical Mexico.

Oriolus niger, [Molothrus aeneus (Wagler 1829) or Dives dives (Deppe 1830)]; 1:169.

Oriolus dominicensis, [Icterus prosthemelas (Strickland 1850)]; 1:176. Mexico and the island of Santo Domingo. (The lumping of the forms $I$. dominicensis and I. prosthemelas within I. dominicensis as possibly conspecific lasted until quite recently [AOU 1998]).

Oriolus castaneus, [Icterus spurius (Linnaeus 1766)]; 1:174, [MNCN]. Tropical Mexico.

Oriolus xanthornus, [Icterus pustulatus (Wagler 1829)]; 1:166. Tropical fields near Cuernavaca [Morelos].

Oriolus occidentalis, [Icterus graduacauda (Lesson 1839)]; 1:172. Tropical Mexico. (Describes the female separately).

Oriolus capensis, [Icterus sp.]; 1:177. Female or immature. Fields near Guadalajara [Jalisco] and the Cape of Good Hope. (Clearly, the authors confuse this species with an African Oriolus).

Oriolus viridis, [Icterus sp.]; 1:184. Female or immature. Tropical Mexico. 
Oriolus cristatus, [Cacicus melanicterus (Bonaparte 1825)]; 1:170. Mexico.

FRINGILLIDAE

Tanagra minuta, [Euphonia hirundinacea (Bonaparte 1838)]; 2:144. Juvenile male. Hueytlalpan [Puebla].

Loxia mexicana, [Carpodacus mexicanus (Müller 1776)]; 2:107, [H]. Mexico.

Fringilla rubra, [Carpodacus mexicanus (Müller 1776)]; 2:155, [H]. Mexico.

Loxia curvirrostra, [Loxia curvirostra (Linnaeus 1758)]. [Image only (MNCN)]. (The painting and background depicted suggest that this specimen may have come from the expedition's outing to the Pacific Northwest of North America).

Loxia subfurca, [Carduelis psaltria (Say 1823)]; 2:105, [H]. Female. Fields near Toluca [Estado de México].

Loxia parvula, [Carduelis psaltria (Say 1823)];

2:103, $[\mathrm{H}]$. Fields near the Valle de México.

Certhia coccinea, [Vestiaria coccinea (Forster 1780)]; 1:289, [H]. Hawaii.

\section{DISCUSSION}

This study comprises the result of compilation of fragmentary information, specifically, the specimens, paintings, and manuscripts generated by the naturalists of the Real Expedición Botánica. Key in this process has been the recent rediscovery of the expedition's ornithological manuscripts (Puig-Samper and Zamudio 1998) as well as at least some of its paintings at the Hunt Institute and the Museo Nacional de Ciencias Naturales (White et al. 1998).

Its specimens, however, remain lost or misplaced. Our searches for the specimens included all of the large European collections that might be expected to hold them (i.e., Museo de Ciencias Naturales in Madrid, Muséum Nationale d'Histoire Naturelle in Paris, and the Natural History Museum in Tring). These specimens, most probably in the form of lifelike mounts for exhibition, would be the last piece of the puzzle to permit definitive identification of the birds collected.

We thus present most of the picture of the expedition's ornithological findings in this paper. The expedition manuscripts are remarkably complete, in that hundreds of Mexican bird species are treated in detail. The paintings are more fragmentary but help considerably in confirming the identifications in the text. Although some identifications may be erroneous owing to our (and possibly Mociño's) limited facility with Latin, we are confident in the great majority of the identifications presented herein.
Provenance of the text and paintings. It is quite clear that the text represents descriptions based on preserved specimens. Although this assertion may seem trivial and difficult to prove, the detail in the manuscripts doubtless reflects careful study of specimen material. For example, the detailed description of the plumage and soft parts of Falco gemebundus (presently Parabuteo unicinctus) would have been possible only through careful study of specimens. Most of the paintings also appear to be based on specimen material, as the positioning of many of the depicted birds is typical of older mounted specimens. Those same positions are repeatedly found when multiple paintings exist for a species (e.g., Charadrius himantopus, presently Himantopus mexicanus). Also, some of the paintings show errors in iris color (e.g., Corvus caeruleus, presently Aphelocoma unicolor), which may suggest glass eyes in mounted specimens. These points coincide with the records of the expedition, which indicate that ornithological specimen material was collected, prepared, and sent to Spain (McVaugh 1977, Bernabéu-Albert et al. 2000).

Given the misadventures of both manuscript and paintings, one may wonder whether they refer to the same specimens. The late 1700 s to early 1800 s was a period of considerable instability in nomenclature of New World birds (Walters 2003). In fact, many species listed in the manuscripts had yet to be described formally and scientifically; as such, close coincidence between the nomenclature used in the paintings with that in the text would be unexpected if they had not been developed by the same researchers looking at the same specimen material. Indeed, we noted such close correspondence - for example, Columba cinerea is a name in the text that appears to be a novel designation for Claravis pretiosa, and the same name was used to label the painting of this species. In general, the great majority $(\sim 96 \%)$ of the Hunt Institute's 83 paintings have names assigned that coincide with names in the text. Such coincidence is not seen in the Museo de Ciencias Naturales paintings, where a larger proportion $(21 \%)$ of names are not found in the text, suggesting that the two sets of paintings may have been separated from each other and the manuscripts at an early date.

In fact, only six cases $(7 \%)$ were observed in which the identification in the text and in the 


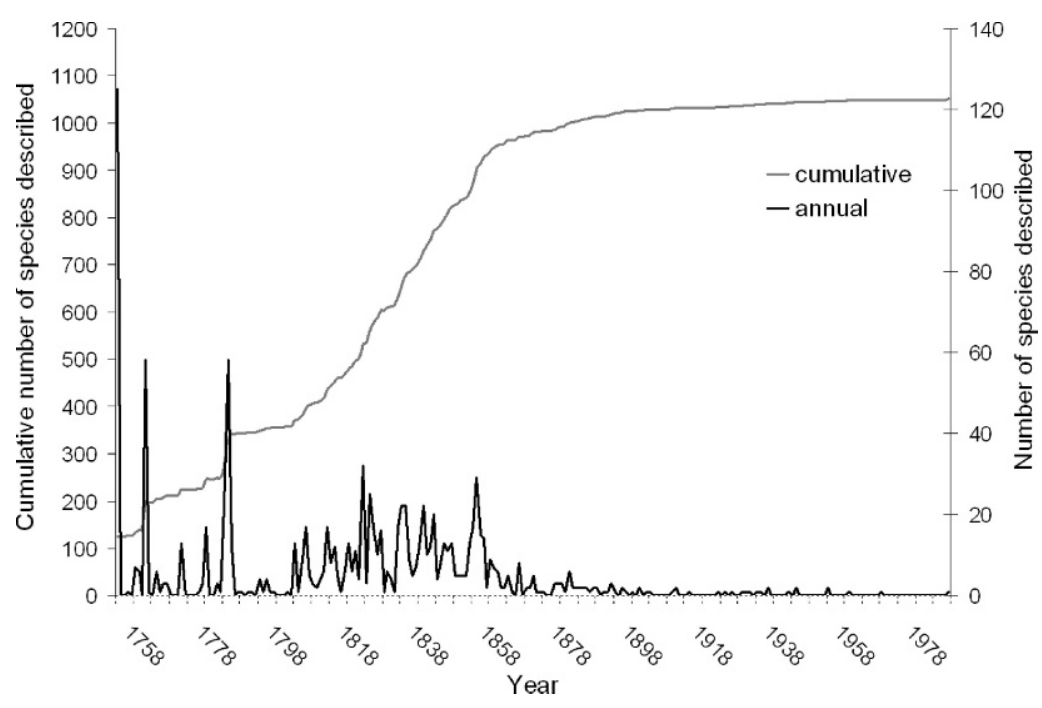

FIGURE 3. Accumulation of described Mexican bird species (AOU 1998) since the original work of Linnaeus. Shown are the cumulative number of species described and the number of species described per year.

corresponding painting were noncoincident. Given the names assigned, the paintings appear to have been misidentified. For example, in the description of Certhia minuta, the description in the text is of Thryomanes bewickii, whereas the painting is of the markedly different Cyanerpes cyaneus - curiously, of the latter species, we see no text entry, in spite of its spectacular coloration and common occurrence in lowland tropical areas. We also noted that many paintings had been assigned multiple identifications or that the original identifications had at times been corrected. We suspect that the few disagreements were caused by erroneous "corrections" by subsequent investigators or that there were errors in detail by the original ornithologists of the expedition. As such, it is likely that the paintings and text were identified by the same group of investigators studying the same group of specimens.

Novelty of the Mociño material. The Real Expedición Botánica occurred very early in the application of Linnaean ideas in ornithology. As mentioned previously, nomenclature was unstable in the late 1700s and early 1800s and varied considerably, particularly with respect to generic allocations. What is more, the main pulse of description of Mexican birds did not occur until the middle $19^{\text {th }}$ century (Fig. 3) indeed, most $(62 \%)$ of the species treated in the Mociño volumes and identifiable by us were still to be described formally when Mociño was working.

Mociño apparently understood that his material included species that were new to science. The distinctive Mexican endemic species Picoides stricklandi, Toxostoma ocellatum, Campylorhynchus jocosus, and Granatellus venustus were not described until after 1850 , yet they were represented in the collections. Although some of Mociño's identifications were erroneous, they represented a best guess given the limitations of the literature that he had available, the limited collections of comparative material that were available, and the incomplete catalogs that had been published at the time. For example, Ramphastos sulphuratus was not described until 2-3 decades later (Lesson 1830); Mociño thus referred his specimen to $R$. picatus, a species described from Brazil in the Sistema Naturae of Linnaeus (1766), and a name no longer considered valid (Peters 1930). Similarly, for the description of Quiscalus mexicanus, Mociño assigned the Linnaean name Corvus senegalensis, from Senegal-we are uncertain of the true identity of that taxon, but it may refer to the African corvid Ptilostomus afer, also black with a long tail. Although modern biogeography would consider this identification to be ridiculous, it was the best solution at the time. For unknown reasons, Mociño did not present formal descriptions of 
his new species, even though he knew that he had dozens at hand.

Mociño clearly had access to the Systema Naturae of Linnaeus (1766; e.g., for Corvus senegalensis) and the works of Gmelin (1788; e.g., for Alcedo americana), or at least to some compilation of those works. Given some nomenclatural and distributional notes (e.g., references to Africa and Asia localities), he probably also had the Ornithologiae of Brisson (1760) and the works of Francisco Hernández (1651). Nevertheless, it is difficult to trace the origin of other names to other sources, and some may have been assigned by Mociño - indeed, had these manuscripts been published, these names would be valid from a nomenclatural perpective. Almost $70 \%$ of names applied by Mociño correspond to taxa to be described later by other authors, particularly William Swainson and William Bullock (1827), Charles Lucien Bonaparte (1825), and Wilhelm Deppe (1830, in Stresemann 1954), among others, from 1808-1914.

It is also clear that most identifications were not made either in the field or in Mexico awaiting shipment to Spain. Rather, this material likely remained unstudied until Mociño went to Madrid in 1804. After Martín Sessé died on 4 October 1808, Mociño continued working, although under difficult conditions - from 1811 on, he dedicated his time to the study of the materials that had been accumulated, collaborating with Pablo de la Llave, a Mexican botanist who also had experience in ornithology (Eisenmann 1959, Maldonado-Polo 1995, Real Jardín Botánico 2005) and who later became the Director of the Real Gabinete de Historia Natural and would describe the Resplendent Quetzal, which he dedicated to Mociño (Pharomachrus mocinno, de la Llave 1832). Other paintings, curiously, appear to have been studied by later workers who may have revisited Mociño's work, as can be noted by different handwriting and notes in French in the illustrations.

Completeness of the inventory. Based on Mociño's manuscripts as historical documents, questions emerge from his ornithological results. One question relates to the sites where the expedition worked and collected birds-how complete was its inventory of local avifauna? The expedition lasted approximately 12 years (McVaugh 1977), with impressive geographic coverage for the time. Omitting sites outside of
Mexico (e.g., Hawaii and Alaska), collected birds came from sites as diverse as Los Tuxtlas in southern Veracruz, Upper California, Guerrero, Nayarit, and Sinaloa. Most of the material came from central Mexico, particularly near Mexico City. It is also known that, during the trips to Mazatlán and San Blas, Mociño and the painter Echeverría embarked on the ships of the expedition led by Alejandro Malaspina (González-Claverán 1988), which may have generated specimens and paintings that may form part of the information summarized herein.

Given the ecological and geographic diversity covered, and only 177 species collected, it is clear that the inventory of Mexican birds was incomplete. Species richness of birds at each of the tropical sites where the expedition worked (Los Tuxtlas, Nayarit, and Guerrero) would have been 150-200 species, and the avifauna across these sites would have been quite different, with relatively little overlap. Avifauna at sites in the interior of Mexico, which are relatively poorer, still would have held 100-150 species. As such, the avian collections of the expedition did not provide a complete panorama of the birds of Mexico, but rather represent occasional collections at particular sites. Nonetheless, given the time period, the manuscripts from the Real Expedición Botánica present a surprisingly complete work. It equals or betters the contributions that would appear decades later from Swainson and Bullock (Swainson 1827), Lafresnaye (1844), and Sclater (1857). In fact, given its detail and careful illustration, this body of work stands out in American ornithology and ranks with the works of Hernández (1651), Catesby (17311743), Audubon (1827), Spix (1824), and Grayson (1986).

Mociño's text includes numerous interesting records of Mexican birds. None is so extreme as not to be credible, but each is interesting and may suggest distributional changes through time. As a consequence, we summarize these special records, with our comments following the species names found in the text:

Phalacrocorax auritus, near Acapulco. This species, carefully described in the text as having an orangereddish face, is not known to occur on the Pacific coast of southern Mexico.

Anhinga anhinga, on Lake Texcoco. This species is of coastal lowland distribution at present and is not known to occur broadly in the Interior, but $19^{\text {th }}$ 
century records also place it in central Mexican lakes (Herrera 1891).

Cochlearius cochlearius, on Lake Tixtla and Lake Texcoco. Similar to the previous species, this species is presently restricted largely to coastal lowland areas; these records suggest that it may have been more broadly distributed previously.

Grus canadensis, on lakes in the Valle de México. This species has been eliminated from much of its distribution in central Mexico, although some evidence of its possible breeding in the Valley of México has been discovered (Martín del Campo 1944).

Bolborhynchus lineola, in Huauchinango and la Huasteca. This parrot species is restricted to cloud forests of eastern Mexico and Central America but at present is known only northward to Oaxaca (Binford 1989, Navarro et al. 1991). These records suggest that it may range north to the mountains of northern Puebla (Gómez de Silva 2003).

Granatellus venustus, near Huasatepeque. This species is restricted to the coastal lowlands of the Pacific slope of Mexico. This record suggests that it may range rarely into the Balsas Depression in Morelos (Rowley 1962).

Quiscalus palustris. Although indicated only in the form of a painting, this record is important in that it places both this species and the related $Q$. mexicanus in the Valley of México contemporaneously. It has also been suggested that $Q$. mexicanus was introduced into the Valley by the Aztecs, who maintained a large aviary (Christensen 2000).

These records all fall within the possibilities of occurrence of each species but are nonetheless intriguing. For example, records of Anhinga, Cochlearius, and Grus in the interior of Mexico, as well as the records of the extinct Quiscalus palustris, are corroborated by 19th century records recently presented (Peterson and Navarro-Sigüenza 2006). None of the three presently occurs in the region.

In conclusion, the ornithological results of the Real Expedición Botánica, were they to have been published early in the $19^{\text {th }}$ century, would have constituted a stellar example of inventory and analysis of bird fauna. Mociño's manuscripts present an impressive level of detail and scientific commentary, and the information they contain is consistent and mostly complete. For various reasons, however, probably more related to history and politics than to science, the ornithological findings of the Real Expedición Botánica were never published. Indeed the manuscript was considered lost for more than 200 years. It is worth mentioning that the botanical results also were lost or neglected, as their text was not published until 1887 (Lozoya 1984), and some packets of herbarium specimens were not unpacked and curated until recent decades (Bernabéu-Albert et al. 2000).

Mociño was hired in 1790 by the viceroy of New Spain, Juan Vicente de Güemes Count of Revillagigedo, to join the team of naturalists that would tour North America, with the special charge of dissecting and preparing birds (Bernabéu-Albert et al. 2000). However, his scientific interests clearly went farther afield than this job, and his contributions, published or not, represent a level of quality of science that far exceeds that of his time. Being such a careful and meticulous scientist, and for his now-appreciated writings on Mexican birds, José Mariano Mociño Suárez de Figueroa, although almost completely forgotten, should now be recognized as the brightest star of the earliest periods of Mexican ornithology.

\section{ACKNOWLEDGMENTS}

Helpful comments on the manuscript were provided by Erick García-Trejo, Livia León, Luis Antonio Sánchez, and two anonymous reviewers. Alejandro Gordillo, Fanny Rebón, Hernán Vázquez, and Luis Antonio Sánchez assisted with certain important details and data. We thank Eric Pasquet (Paris); Josefina Barreiro (Madrid); Robert Prys-Jones, Mark Adams, and Michael Walters (Tring) for permitting access to and facilitating our work in collections and libraries under their care. Thanks also to the Hunt Institute for Botanical Documentation for permission to reproduce the painting in Fig. 2; this was kindly facilitated by Robert W. Kiger and James White. Financial support for this research was provided by CONACYT (R27961), CONABIO (V009), Fondo Sectorial SEMARNAT-CONACyT (0265) and the "Cátedras de Excelencia" program of CONACyT (to ATP).

\section{LITERATURE CITED}

AMERICAN ORNITHOLOGISTS' UNION. 1998. Checklist of North American Birds. 7th ed. American Ornithologists' Union, Washington, DC.

Arias Divito, J. C. 1968. Las expediciones científicas españolas durante el siglo XVIII: expedición botánica de Nueva España. Ediciones Cultura Hispánica, Madrid, Spain.

Audubon, J. J. 1827. The birds of America, from original drawings. Published by the author, London.

BArreiro-Rodríguez, J. 1987. Catálogo de las láminas del Museo Nacional de Ciencias Naturales, p. 345-351. In Real Jardín Botánico: la Expedición Botánica a Nueva España 17871803. Biblioteca V Centenario, Madrid, Spain.

Bernabéu-Albert, S., P. Blanco Fernández de Cayela, H. M. Burdet, E. Cobos Sanpedro, I. H. W. Engstrand, E. García Guillén, J. L. Maldonado, R. McVaugh, M. Puig-SAMPer, 
AND G. ZAMUDIO. 2000. El águila y el nopal: la expedición de Sessé y Mociño a Nueva España (1787-1803). Lunwerg Editores, Madrid, Spain.

BINFORD, L. C. 1989. A distributional survey of the birds of the Mexican state of Oaxaca. Ornithological Monographs 43.

Bonaparte, C. L. 1825. Descriptions of two new species of Mexican birds. Journal of the Academy of Natural Sciences of Philadelphia 4:387-390.

BRISSON, M. J. 1760. Ornithologia, sive synopsis methodica sistens avium divisionem in ordines. C. J. B. Bauche, Paris, France.

CAmelo, R., A. Fernández-Águila, And B. A. VÁZquez-RomÁn. [ONLINE]. 2007. Nueva España: siglos XVI y XVII, Map II.2.2. In Atlas nacional de México. Instituto de Geografía, Universidad Nacional Autónoma de México, México, <www.igeograf.unam.mx/instituto/ publicaciones/atlas_nacional.htm $>(2$ September 2007).

CATESBY, M. 1731-43. The natural history of Carolina, Florida and the Bahama Islands. Vols. 1-2. London.

Christensen, A. F. 2000. The fifteenth- and twentieth-century colonization of the Basin of Mexico by the Great-tailed Grackle (Quiscalus mexicanus). Global Ecology and Biogeography 9:415-420.

DE LA Llave, P. 1832. Memoria sobre el Quetzaltototl, género nuevo de aves. Registro Trimestre $1: 48$.

Dickinson, E. [ED.]. 2003. The Howard and Moore complete check-list of the birds of the world. Princeton University Press, Princeton, NJ.

EISENMANN, E. 1959. The correct specific name of the Quetzal, Pharomachrus mocinno. Auk 76:108.

Escalante, P., A. G. NAvarro, And A. T. Peterson. 1993. A geographic, historical and ecological analysis of land bird diversity in Mexico, p. 281-307. In T. P. Ramamoorthy, R. Bye, A. Lot, and J. Fa [EDS.], Biological diversity in Mexico: origins and distribution. Oxford University Press, New York.

Gmelin, J. F. 1788. Caroli a Linné...Systema naturae per regna tria naturae, secundum classes, ordines, genera, species, cum characteribus, differentiis, synonymis, locis. Vol. 1, 13th ed. G. E. Beer, Leipzig.

Goldman, E. A. 1951. Biological investigations in Mexico. Smithsonian Miscellaneous Collections 115:1-476.

Gómez de Silva, H. [ED.]. 2003. Mexico. North American Birds 57:123-129.

GómEZ-VÁzQuez, H. 1997. Viajeros naturalistas y científicos novohispanos en el siglo de las luces. México Desconocido 240:32-42.

GonzÁlez Claverán, V. 1988. La expedición científica de Malaspina en Nueva España 17891794. El Colegio de México, Mexico City.

Grayson, A. J. 1986. Birds of the Pacific Slope: one hundred fifty-six bird portraits painted in California and Mexico, 1853-1869. Arion Press, San Francisco, CA.
HeRnÁndez, F. 1651. Nova plantarum animalium et mineralium Mexicanorum historia. Rome, Italy.

Herrera, A. L. 1891. Apuntes de ornitología: la migración en el Valle de México. La Naturaleza, 2nd series 1:165-189.

Howell, S. N. G., AND S. WebB. 1995. A guide to the birds of Mexico and northern Central America. Oxford University Press, New York.

LAFRESNAYe, F. 1844. Oiseaux nouveaux du Mexique. Revue Zoologie 7:41-43.

LAMAS, G. 1986. Ilustraciones inéditas de lepidópteros mexicanos de la expedición de Sessé y Moziño (1787-1803). Revista Sociedad Mexicana de Lepidopterología 10:27-34.

Lesson, R. P. 1830. Traité d'ornithologie. Vol. 3. Paris, France.

LinNAEUS, C. 1766. Systema naturae per regna tria naturae, secundum classes, ordines, genera, species, cum characteribus, differentiis, synonymis, locis. 12th ed., Vol. 1. Stockholm.

LONG-LOST SESSÉ AND MOCIÑO ILlUSTRATIONS ACQUIRED. 1981. Bulletin of the Hunt Institute for Botanical Documentation 3:1-3.

LozoyA, X. 1984. Plantas y luces en México: la Real Expedición Científica a Nueva España (17871803). Ediciones del Serbal, Barcelona, Spain.

MAldonAdo-Polo, L. 1995. La flora de Guatemala de José Mociño. Ediciones Doce Calles, Madrid, Spain.

MARTín Del CAMPo, R. 1944. Huevos subfósiles de grulla en el Valle de México. Anales Instituto de Biología de la Universidad Nacional Autónoma de México 15:313-318.

McCoy, C. J., AND O. A. Flores-Villela. 1985. Amphibians and reptiles of Sessé and Mociño expedition: a lost chapter in Mexican herpetology. Annals of the Carnegie Museum 54:189-193.

McVaugh, R. 1977. Botanical results of the Sessé and Mociño expedition (1787-1803). I. Summary of excursions and travels. Contributions from the University of Michigan Herbarium 11: 97-195.

Mearns, B., And R. Mearns. 1998. The bird collectors. Academic Press, London.

NAvarro, A. G. 1989. La sistemática ornitológica en México: posibilidades y limitaciones. Revista Especial Ciencias 3:96-102.

Navarro, A. G., M. G. Torres Chávez, And P. Escalante. 1991. Catálogo de Aves. Serie Catálogos Museo de Zoología 4:1-300.

Peters, J. L. 1930. The identity of the toucans described by Linnaeus in the 10th and 12 th editions of the Systema Naturae. Auk 47:405-408.

Peterson, A. T. 1998. The distribution and type locality of the extinct Slender-billed Grackle, Quiscalus palustris. Bulletin of the British Ornithologists' Club 118:119-121.

Peterson, A. T., And A. G. Navarro-Sigüenza. 2006. Hundred-year changes in the avifauna of the Valley of Mexico, Distrito Federal, Mexico. Huitzil 7:4-14. 
Phillips, A. R. 1986. The known birds of North and Middle America, Part 1: Hirundinidae to Mimidae; Certhiidae. A. R. Phillips, Denver Museum of Natural History, Denver, Colorado.

Puig-SAmper, M. A., AND G. ZAMudio. 1998. Un manuscrito inédito de la Real Expedición Botánica a Nueva España (1787-1803) sobre ornitología mexicana. Asclepio 50:251-254.

REAL JARdín BotÁNICO. [ONLINE]. 2005. Expedición al Virreinato de Nueva España: tras las huellas de F. Hernández (1787-1803). <www.rjb.csic.es/ historia_nuevaespana.php $>$ (2 September 2007).

RIDGWAY, R., AND H. FRIEDMANN. 1901-1950. The birds of North and Middle America. United States National Museum Bulletin 50 Collection.

Rowley, J. S. 1962. Nesting of the birds of Morelos, Mexico. Condor 64:253-272.

SAlvin, O., AND F. D. Godman. 1879-1904. Biologia Centrali-Americana, Aves. Vols. 1-4. Taylor and Francis, London.

SCHIFTER, H. 1996. Von Ferdinand Deppe und Christoph Julius Wilhelm Schiede in Mexiko gesammelte Vögel im Naturhistorischen Museum Wien. Mitteilungen aus dem Zoologischen Museum in Berlin 72:3-25.

SClATER, P. L. 1857. List of additional species of Mexican birds obtained by M. A. Sallé from the environs of Jalapa and San Andres Tuxtla. Proceedings of the Zoological Society of London 25:201-207.
Sessé, M., AND J. M. MociÑo. 1893. Plantae Novae Hispaniae. 2nd ed. Secretaría de Fomento, México.

Sessé, M., AND J. M. Mociño. 1894. Flora Mexicana. 2nd. ed. Secretaría de Fomento, México.

Sibley, D. A. 2000. The Sibley guide to birds. Alfred A. Knopf, New York

SPIX, J. B. VON. 1824. Avium species novæ, quas in itinere per Brasiliam annis MDCCCXVIIMDCCCXX. Munich.

StREsSEMANN, E. 1954. Ferdinand Deppe's travels in Mexico, 1824-1829. Condor 56:86-92.

SWAINSON, W. 1827. A synopsis of the birds discovered in Mexico by W. Bullock, F.L.S., and H.S., Mr. William Bullock. Philosophy Magazine (New Series) 1:364-369, 433-442.

U.S. Geological Survey. [Online]. 2006. HYDRO1k Elevation Derivative Database. <http:// edcdaac.usgs.gov/gtopo30/hydro/index.asp > (20 August 2006).

WALTERS, M. 2003. A concise history of ornithology. Yale University Press, New Haven, CT.

White, J. J., R. McVaugh, And R. W. Kiger. 1998. The Torner Collection of Sessé and Mociño biological illustrations. Carnegie Mellon CD Press, PA.

Zamudio, G., AND A. ButAndA. 1999. Humboldt y la botánica americana. Revista Ciencias 5556:36-43. 